\title{
REVIEW
}

\section{Recommendations for palliative and bereavement care in the NICU: a family-centered integrative approach}

\author{
C Kenner ${ }^{1,2}$, J Press $^{3}$ and D Ryan ${ }^{4}$
}

Technological advances have increased our ability to detect a life-threatening, life-limiting or lethal problem early in pregnancy, leaving parents months to anticipate a death or a prematurely born infant. Babies can also be born with unanticipated problems that could lead to death. In either scenario, perinatal palliative care should be offered as a strategy for family support. Since the preponderance of professional training focuses on saving lives, many health professionals are uncomfortable with palliative care. This article's purpose is to define best practices for the provision of family-centered perinatal and neonatal palliative care and provision of support to bereaved families experiencing anticipated and unanticipated life-limiting conditions or death of their infant. An overview of core concepts and values is presented, followed by intervention strategies to promote an integrated familycentered approach to palliative and bereavement care. The concluding section presents evidence-based recommendations.

Journal of Perinatology (2015) 35, S19-S23; doi:10.1038/jp.2015.145

\section{INTRODUCTION}

Palliative and bereavement care date back for decades. Dame Cicely Saunders used the term 'hospice care' in 1948 to describe specialized care of the dying. ${ }^{1}$ In 1997 , the emphasis was on quality of life during the dying process. ${ }^{1}$ As technology pushed the age of viability for newborn infants downward, smaller, more medically fragile babies started surviving. Although survival for those infants $<1000 \mathrm{~g}$ has improved, the overall neonatal mortality rate in 2011 was 4.04 per 1000 live births. ${ }^{2}$ Technological advances have also made it easier to detect a life-threatening, lifelimiting or lethal problem early in pregnancy, giving families the chance to make an informed decision about continuing the pregnancy and planning for the birth process and beyond.

Perinatal palliative care gained prominence in the early 2000's, when Catlin and $\mathrm{Carter}^{3}$ highlighted the need for a coordinated plan for delivering neonatal palliative care. Catlin and Carter recognized that palliative and bereavement care are often provided in a fragmented approach, with many disciplines operating in their own 'silos', each offering advice without knowing what else has been said to overwhelmed families. Unfortunately, the same fragmentation occurs in many neonatal intensive care units (NICUs) today, in spite of the fact that the American Academy of Pediatrics has outlined numerous practices for pediatric palliative care. ${ }^{4,5}$

Palliative care can now be offered to parents as an alternative to termination of pregnancy when a fetus is diagnosed with a lifelimiting condition. It is a viable treatment option for babies born at the edge of viability, ${ }^{6}$ diagnosed with life-limiting conditions at birth or who become critically ill during a NICU stay and are not responding to aggressive medical management. ${ }^{7}$ Decisions to not initiate or to withdraw intensive care from a baby can be both ethically challenging ${ }^{8,9}$ and morally distressing. ${ }^{10-12}$

Arriving at such a decision through an interdisciplinary, familycentered team approach can shorten futile intensive care and minimize suffering of both babies and their parents. ${ }^{13}$ Several studies have shown that providing intensive care to babies with pre- or postnatally diagnosed life-limiting conditions does not prolong life. ${ }^{14,15}$ Hence, palliative care serves as an alternative to intensive care, continuing to provide quality care and grief support while honoring both the baby and the family. Identification of best practices and evidence-based guidelines to help health professionals provide the best possible palliative care in a compassionate manner is needed. This article's purpose is to define best practices for the provision of family-centered palliative care for fetuses and infants and to support bereaved families experiencing anticipated and unanticipated life-limiting conditions or death of their baby.

\section{CORE CONCEPTS AND VALUES}

Palliative care is an approach that improves the quality of life of patients facing life-limiting conditions, and their families, through the prevention and relief of suffering by means of early, impeccable assessment and treatment of pain and other physical, psychosocial and spiritual issues. ${ }^{16}$ For this paper, palliation will refer to comfort care for the neonate who is facing a life-limiting condition, whether or not death is expected, and supportive care for the baby's family. This care promotes quality of life and includes the family as an integral member of the health-care team. ${ }^{16}$ The setting for the care may be the acute care hospital, an ambulatory care facility in the community or the home. ${ }^{16}$ Palliative care is still an option that is offered unevenly and too late in many instances, and rarely delivered in an integrated approach. Bereavement care focuses on the grief process by providing interventions that support families after a fetal, neonatal or infant loss, with the goal of decreasing feelings of sorrow, psychosocial stress and social isolation. ${ }^{17}$ Grief support is encompassed in palliative care.

\footnotetext{
${ }^{1}$ School of Nursing, Health and Exercise Science, The College of New Jersey, Ewing, NJ, USA; ${ }^{2}$ Council of International Neonatal Nurses, Yardley, PA, USA; ${ }^{3}$ Perinatal Bereavement Services, Crouse Hospital, Syracuse, NY, USA and ${ }^{4}$ Nurse Education Program, Elmira College, Elmira, NY, USA. Correspondence: Dr C Kenner, School of Nursing, Health and Exercise Science, The College of New Jersey, PO Box 7718, Ewing, NJ 08628-0718, USA.

E-mail: kennerc@tcnj.edu

Received 4 September 2015; accepted 18 September 2015
} 
Family is defined as a constellation of people who are related by birth, adoption, marriage or those individuals the parents designate as part of their family unit. The family constellation is crucial to provide individualized care that embraces a familycentered approach. Family-centered care is defined as delivery of services that revolve around the incorporation of the family in the development and implementation of interventions; it represents a family-professional partnership. ${ }^{18}$ The family is an integral part of the health-care team, and both work together to plan and implement palliative and/or bereavement care. Core values or key concepts that support palliative and bereavement care are compassion, commitment to the delivery of palliative/bereavement care, dignity, integrity, respect, information-sharing, parental decision-making and teamwork.

Families may experience an anticipated or unanticipated loss. Although each loss requires a slightly different approach, the common guiding principles include providing family-centered, compassionate and individualized care. The family must be included in the decision-making process to the degree they deem themselves able to participate, guided by their understanding of their child's best interest. ${ }^{4}$

\section{UNANTICIPATED LOSSES}

When a family experiences an early loss such as from a miscarriage, ectopic pregnancy, fetal demise or the birth of an extremely premature infant, there is no opportunity to plan or prepare for the death. Perinatal loss needs to be recognized as a unique type of bereavement, and even early fetal losses or stillbirths will require bereavement care. Such losses disrupt a significant life milestone (pregnancy/birth/infancy) and can cause isolation from peers, inner conflict for the grievers and a reorganized sense of self-esteem. ${ }^{19} \mathrm{~A}$ child's death is unlike any other loss, profoundly affecting parents individually and as a couple, as well as the family system and society. ${ }^{20}$ The family is likely to be in shock as they are forced to adjust from having the infant they anticipated to one born dying. In cases of unanticipated loss, palliative and comfort care may be offered as indicated. ${ }^{21}$

The interprofessional team needs to be fully informed of parents' choices so that care is coordinated and the family's wishes are carried out. If a baby in the NICU is expected to die, then providers need to determine the family's wishes for resuscitation of their infant, and whether they wish to sign a DNR (Do Not Resuscitation) order. Many families are unwilling or unable to face a decision that may make them feel as though they are responsible for ending their baby's life. They may be more accepting of a plan to not resuscitate if asked to agree to AND (Allow a Natural Death). The term AND is used interchangeably with DNR and refers to the desire not to aggressively resuscitate an infant or intervene if a code occurs. ${ }^{22}$

\section{ANTICIPATED LOSSES}

Advances in genetics and technology enable many families to know well in advance that their fetus has a potentially life-limiting condition. Prenatal genetics refers to the use of genetic testing and counseling during a pregnancy. Non-invasive genetic screening, offered very early in pregnancy, has became more accessible. Abnormalities in prenatal screening tests can lead to further evaluation, including both ultrasound and more definitive genetic testing to provide further information about the cause of fetal anomalies. This process can afford parents time to make decisions early in the pregnancy, such as whether they want to pursue medical interruption of the pregnancy, medical interventions such as fetal or neonatal surgery, or preparation for a fetal or neonatal death. 23

Antenatal consults should occur in the context of a family meeting with the specialists from both obstetrics and neonatology, the palliative care team and support provided by a NICU mental health professional. A coordinated, integrated team approach is needed so that the family gets a consistent message indicating that they are the architects of this plan to the degree they wish. Results of genetic and other diagnostic evaluations, and information about the condition of the fetus and its prognosis should be shared among the group. Options for palliative care as an alternative to termination of pregnancy can be presented to the family, and a plan of action created according to the family's wishes.

Anticipatory grief is a term that is used when a death is anticipated or expected; it starts when the family recognizes that the outcome will not be what they anticipated. They experience the loss of their envisioned future as a family. Often, their usual social support system is inaccessible due to discomfort and to the absence of many cultural norms for acknowledging an anticipated loss. The family loses the rites of passage that make the baby more real and that facilitate bonding within the family unit such as having a baby shower or homecoming party.

A death that is anticipated before birth affords the family the opportunity to work with the health-care team to develop both a birth plan, which can specify their desires for how they want the birth to evolve, and a palliative care plan. The family's participation in making these plans helps to provide them with a sense of control, and the plan honors their choices for their baby. When the birth occurs, the plans should be followed regardless of which health professionals are present at the time.

In either anticipated or unanticipated losses, efforts should be made to include the families as a part of the decision-making process without overwhelming them. Families need to be provided options for memory-making, and asked whether there is a ritual that is important to their culture or belief system that they wish to see happen. Parents may choose to keep a lock of hair, handprints and/or footprints, clay models of the newborn's hands or feet, photos, a receiving blanket, and special boxes or envelopes for keepsakes. The key is to ask the family what they want while realizing they may not know. A plan for the death is just as important as a birth plan and should include the family's plans for organ donation when applicable. ${ }^{24,25}$

\section{DURING THE MOTHER'S AND BABY'S HOSPITALIZATION}

The goal for hospitalization at the baby's birth is for the family to be as prepared mentally, physically and spiritually as possible. Specific information should be given to the parents to address their questions and concerns both before and after the baby's birth. It is important to have a structured bereavement program that is available to staff at all times; written policies and standards for this program should be available to all hospital personnel.

If the mother is in one hospital after giving birth, and the baby in another, then information may be given to the father (or other family member) before the mother, leaving her with unanswered questions. Thus, all attempts should be made to keep the mother informed. It is also important to recognize that parents' understanding of their baby's condition may differ depending on the information they have received, their anxiety levels, their prior experiences with loss and the way the information is provided.

A mother who has given birth to a baby who has died or who is expected to die should be cared for with the utmost sensitivity. All staff that could potentially enter her room should be made aware of her loss. Mothers may even be offered the option to receive their postpartum care on a unit other than the usual mother/ baby unit.

Although providing comfort with dignity, parents must be asked whether they have personal preferences, and staff should not assume that all of the family's reactions are culture based. Giving families mementos or photographs related to their baby's hospital stay provides them with options for remembering their 
baby, although these may not be acceptable in some cultures. Photography is an important remembrance to some families, as photographs provide visual proof of the reality their baby's life and are valuable in the mourning process. ${ }^{26}$ Photographs should be free to families and can be provided through a volunteer organization of professional photographers, ${ }^{27}$ a contracted photography company or families may prefer to take their own photographs.

Dressing the baby is important so that the family has a photo or mental picture of their child as a baby and not one that is naked, covered with tubes and wires. Families may choose to dress a baby in special clothes such as Angel Gowns, which are available for free to all hospitals upon request from a parent support organization. ${ }^{28}$ Parents should be offered peer-to-peer support, and those whose baby expires should be offered the opportunity to be paired with a bereavement mentor for such support (see 'Recommendations for peer-to-peer support for NICU parents', this issue). In hospitals without functioning peer support programs, parents should be referred, with their consent, to regional or national parent support organizations that have been evaluated by their NICU staff.

\section{FAMILIES OF MULTIPLES}

When one infant in a set of multiples has died or is actively dying, families face an emotionally complex and volatile situation. Families are grieving while balancing hope for a baby or babies who are still alive. In addition to providing the usual bereavement support, health professionals must also acknowledge that the parents may be conflicted, as these parents are experiencing joy and grief simultaneously. ${ }^{26}$ It is best to photograph multiples together and separately, even if one of the babies is alive, making sure to have a process to differentiate the babies from each other. This further acknowledges the specific grief. ${ }^{26}$

\section{DIFFERENCES BETWEEN FATHERS AND MOTHERS}

Fathers may react differently from mothers to losses. In some cultures, fathers are viewed as the protector; if a baby dies, then the father may feel as though he has failed because he could not protect the family. Mothers may feel that they failed as a woman to produce a healthy child. Men and women may also grieve differently. ${ }^{29}$ The father may return to work and his social network sooner than the mother. He may want to process the grief alone and may be reluctant to share his feelings even with the mother of his child. Mothers often want to talk through their emotions and to process the death. Mothers may be more accepting of support groups and counseling than fathers. Differences in perceptions may never be shared between the parents unless they are encouraged to talk together with guidance from a health professional, clergy or parent support liaison. ${ }^{30}$

\section{DISCHARGE TO HOME BEFORE DEATH}

The time after discharge of the mother and/or baby is an emotionladen time for families. Medical technology used in the home may create a high degree of parental emotional stress. Parents of medically fragile and technology-dependent children have identified role conflict and care burden as sources of stress. ${ }^{31}$ If a family is taking their baby home to die, then their ability to cope should be assessed before discharge, including what support systems are in place and whether palliative and/or hospice care for families and neonates is available in their community.

For babies not expected to survive, plans should be developed for a home death with the participation of the family. Supportive contact for the parents should be available going forward, such as by hospice nurses who come to the home to assist the family with a dying baby, by support groups or by peer mentors that either meet face-to-face or in an online community.

\section{FOLLOWING THE DEATH}

Burial arrangements and final disposition of the infant's remains are important (although difficult) topics to address, and hospital staff, particularly social workers, may open the conversation and provide information to families regarding their options or suggest the family designate someone to investigate services available in their community. Hospital services should be outlined verbally, and written information provided to the parents.

A letter or sympathy card should be mailed to the family 1 to 2 weeks after the loss, signed by the health-care professionals who actually cared for their baby, as an important and personal gesture. The name of a grief counselor or a list of general bereavement support groups/agencies should be provided to the family, if it has not been already.

\section{STAFF EDUCATION}

Staff working with families facing or experiencing a perinatal loss must be educated on how to work with families under extreme stress and how to have difficult conversations. ${ }^{5}$ Several studies have shown that both nurses and physicians feel ill-prepared to have conversations with families regarding end-of-life issues. ${ }^{32,33}$ However, a number of educational and training programs have been developed for medical ${ }^{32,33}$ and nursing students and trainees, faculty and practicing health professionals. ${ }^{34,35}$

\section{STAFF SUPPORT}

Palliative care work is stressful, and caregivers in both Labor and Delivery units and in the NICU are themselves at risk for burnout, compassion fatigue, secondary traumatic stress syndrome and/or post-traumatic stress disorder. ${ }^{36,37}$ It is important that staff have a chance to debrief and gain support when working with perinatal or neonatal loss, and have the opportunity to participate in bereavement interventions such as attending funerals or memorial services. ${ }^{38}$ If caregivers do not get emotional support, then they will have less to give to the families. ${ }^{30}$ Staff can be referred for counseling or obtain support from the pastoral care staff. ${ }^{39,40}$

\section{RECOMMENDATIONS}

1. Parents who lose a baby before, during, or shortly after birth, or later in the NICU should be offered:

a. Anticipatory guidance regarding the grieving process, including how mothers and fathers, and other family members, may grieve differently. ${ }^{29}$ This anticipatory guidance should begin antenatally if a life-limiting diagnosis has been determined. $^{40,41}$

b. Participation in bereavement rituals, including those that meet their spiritual, religious and cultural preferences. These rituals may include a variety of practices and items that will help them remember their baby, such as provision of hand prints, foot prints and photographs. ${ }^{42-44}$ Smooth communication should be facilitated between parents and the staff of the NICU and obstetric units throughout the dying process; a perinatal social worker or other designated person can assist with this. ${ }^{45}$

c. Psychosocial support for all members of the family, including but not limited to grandparents and the baby's siblings.,46

d. Peer-to-peer support and/or referral to community or Internet support organizations (see 'Recommendations for peer-to-peer support for NICU parents', this issue). 
e. Counseling regarding both the physical and psychological considerations of attempting another pregnancy. ${ }^{5}$

f. Post-hospital follow-up in a variety of forms, including individual contact by a staff member at various time periods as well as a conference 4 to 6 weeks after the baby's death to review autopsy and other results that will help parents understand what happened to their baby. ${ }^{47}$

2. When intensive care will not be provided, a collaborative, interdisciplinary approach should be used to provide palliative care including bereavement care to any family whose fetus or neonate is facing a life-limiting condition or imminent death. ${ }^{48}$

3. Physicians and nurse practitioners should follow the guidelines outlined by the American Academy of Pediatrics when engaging in discussions with parents about whether to initiate or continue intensive care for a baby who may not survive; ${ }^{4,6}$ neonatal nurses and perinatal social workers should be included in the decisionmaking process as well. ${ }^{45,49}$ Intensive care should be provided only if it is believed that the baby will benefit from it. The best interests of the baby should be the guiding standard.,

4. In cases where there are disagreements between parents and the health-care team when making end-of-life decisions, particularly around the futility of further care for a baby, a bioethics consult should be obtained and/or the case presented to the hospital Bioethics Committee., ${ }^{40}$

5. Parents whose babies with life-limiting conditions survive to hospital discharge should be offered both practical and psychosocial support to help them prepare for discharge, learn how to both care for and cope with their baby at home, ${ }^{51,52}$ and/or they should be referred to a hospice with experience caring for infants. Their baby's primary care provider, neonatal specialist, medical home or any subspecialists should be fully involved in making and carrying out home care plans and providing follow-up support. ${ }^{5}$

\section{ANTENATAL RECOMMENDATIONS}

Parents anticipating a perinatal loss should be offered:

1. An antenatal interdisciplinary conference to develop the following plans, which should be individualized according to parents' desires and cultural, spiritual and religious beliefs:

a. A birth plan, which should include parents' preferences regarding the conduct of labor, the circumstances surrounding delivery and the care for baby and parents after the baby's birth;

b. A plan for the extent of resuscitation to be performed and whether to allow a natural death; and

c. A palliative care plan as indicated and desired when babies are not expected to survive. This plan should include parents' preferences on where the baby's care will be delivered, how nutrition will be provided and how baby's pain and discomfort will be managed. ${ }^{51,52}$

2. Encouragement to bond with their baby and to create memories throughout the pregnancy whenever feasible, recognizing the barriers parents may face in bonding.

3. Psychosocial support for all members of a family, including grandparents and the infant's siblings.

4. Both practical and psychosocial support to help parents cope with their baby at home when infants with life-limiting conditions survive to hospital discharge. ${ }^{5}$

\section{HEALTH SYSTEM RECOMMENDATIONS}

1. All health professionals who work with pregnant women, neonates and their families should receive education and demonstrate competence in palliative and bereavement care that is appropriate within their scope of practice. This education should include training in how to communicate effectively and empathetically with families. ${ }^{5,21,33}$

2. Policies for palliative care and bereavement practices in any part of a health-care system should be in place and easily accessible to all staff, to ensure a standard of care for all families.

3. Palliative and bereavement care resources in the community should be available to anyone who is providing care to a neonate and family facing a life-threatening condition or imminent death or has experienced a loss, whether or not there is a palliative care team or program at a specific institution.

4. Hospitals should work in partnership with their local organ donation organizations to determine whether specific babies are eligible to become organ donors and to assess, in collaboration with the family, whether organ donation is the right choice for them. ${ }^{5}$

5. Health-care staff who provide palliative and/or bereavement care should receive psychosocial support and engage in selfcare $^{53}$ to enable them to provide optimal care to the families they serve, as well as to prevent burnout, compassion fatigue or secondary traumatic stress. ${ }^{5}$ The perinatal social worker, ${ }^{44} \mathrm{NICU}$ psychologist ${ }^{54}$ or pastoral care staff ${ }^{55}$ can be instrumental in providing this support.

\section{CONCLUSION}

A family-centered, integrated culturally driven plan of care can provide support for families experiencing an anticipated or unanticipated loss. The goal of palliative and bereavement care is to make the families and neonates as comfortable as possible. It is important to engage the family in the plan of care and to recognize that each family is different in how they wish to approach this situation. Palliative and bereavement care must be considered as standard care and treated as an expectation, not as optional additional services. Staff must also be supported, as palliative care and bereavement care are critical but emotionally draining aspects of perinatal/neonatal care. This article presents evidence-based recommendations for perinatal-neonatal, familycentered palliative and bereavement care.

\section{CONFLICT OF INTEREST}

The authors declare no conflict of interest.

\section{ACKNOWLEDGEMENTS}

Many thanks to other participants from the National Perinatal Association Workgroup on Palliative and Bereavement Care for their contributions to this work including Amanda Hedin, Laura Martin and Raja Nandyal. This article has been supported by grants and contributions from: the Wellness Network, Prolacta Bioscience, Division of Neonatology at Loma Linda University School of Medicine, Brenau University, NICU Parent Support at Mercy Hospital in St. Louis, MO, Hand to Hold, Preemie Parent Alliance, Zoe Rose Memorial Foundation, the Rosemary Kennedy Trust and Eden's Garden.

\section{REFERENCES}

1 National Hospice and Palliative Care Organization. History of Hospice Care [Internet]. National Hospice and Palliative Care Organization: Alexandria, VA, 2015 Available from http://www.nhpco.org/history-hospice-care.

2 Mathews T, MacDorman M. Infant Mortality Statistics from the 2010 Period Linked Birth/infant Death Data Set. US Department of Health and Human Services, Centers for Disease Control and Prevention: Washington, DC, USA, 2013, pp 1-26. Report No.: 62(8). Available from http://www.cdc.gov/nchs/data/nvsr/ nvsr62/nvsr62_08.pdf.

3 Catlin A, Carter B. Creation of a neonatal end-of-life palliative care protocol. J Perinatol 2002; 22(3): 184-195. 
4 American Academy of Pediatrics Committee on Fetus and Newborn. Noninitiation or withdrawal of intensive care for high-risk newborns. Pediatrics 2007; 119(2): 401-403.

5 American Academy of Pediatrics Section on Hospice and Palliative Medicine and Committee on Hospital Care. Pediatric palliative care and hospice care commitments, guidelines, and recommendations. Pediatrics 2013; 132(5): 966-972.

6 Cummings J, American Academy of Pediatrics, Committee on Fetus and Newborn. Antenatal counseling regarding resuscitation and intensive care before 25 weeks of gestation. Pediatrics 2015; 136(3): 588-595.

7 Bhatia J. Palliative care in the fetus and newborn. J Perinatol 2006; 26(Suppl 1): S24-S26.

8 Conway A, Molony-Harmon P. Ethical issues in the neonatal intensive care unit. Crit Care Nurs Clin North Am 2004; 16(2): 271-278.

9 Cuttini M, Casotto V, de Vonderweid U, Garel M, Kollee L, Saracci R et al. Neonatal end-of-life decisions and bioethical perspectives. Early Hum Dev 2009; 85(10 Suppl): S21-S25.

10 Carter B, Leuthner S. The ethics of withholding/withdrawing nutrition in the newborn. Semin Perinatol 2003; 27(6): 480-487.

11 Catlin A, Armigo C, Volat D, Vale E, Hadley M, Gong W et al. Conscientious objection: a potential neonatal nursing response to care orders that cause suffering at the end of life? Study of a concept. Neonatal Netw 2008; 27(2): 101-108.

12 Gale G, Brooks A. Implementing a palliative care program in a newborn intensive care unit. Adv Neonatal Care 2006; 6(1): 37-53.

13 Baumann-Holzle $\mathrm{R}$, Bucher $\mathrm{H}$, Maffezzoni M. A framework for ethical decision making in neonatal intensive care. Acta Paediatr 2005; 94(12): 1777-1783.

14 Courtwright $A$, Laughon $M$, Doron $M$. Length of life and treatment intensity in infants diagnosed prenatally or postnatally with congenital anomalies considered to be lethal. J Perinatol 2011; 31: 387-391.

15 Parravicini E, Lorenz J. Neonatal outcomes of fetuses diagnosed with life-limiting conditions when individualized comfort measures are proposed. J Perinatol 2014, 34(6): 483-487.

16 World Health Organization (WHO). WHO Definition of Palliative Care; 2015. Available from http://www.who.int/cancer/palliative/definition/en/ (accessed on 27 July 2015).

17 Forte A, Hill M, Pazder R, Feudtner C. Bereavement care interventions: a systematic review. BMC Palliat Care 2004; 3(1): 3.

18 McGrath J, Samra H, Kenner C. Family-centered developmental care practices and research: what will the next century bring? J Perinat Neonatal Nurs 2011; 25(2): 165-170.

19 Leon I. The psychoanalytic conceptualization of perinatal loss: a multidimensional model. Am J Psychiatry 1992; 149(11): 1464-1472.

20 Rando T. The Unique Issues and Impact of the Death of a Child. Parental Loss of a Child. Research Press Company: Champaign, IL, USA, 1986, pp 5-43.

21 National Association of Neonatal Nurses. Palliative and End-of-life Care for Newborns and Infants. Position Statement \#3063. National Association of Neonatal Nurses: Chicago, IL, 2015. Available from http://www.nann.org/uploads/files/PalliativeCare6_ FINAL.pdf.

22 Breault JL. DNR, DNAR, or AND? Is language important? Ochsner J 2011; 11(4): 302-306.

23 Sobel S, Cowan CB. Ambiguous loss and disenfranchised grief: the impact of DNA predictive testing on the family as a system. Fam Process 2003; 42(1): 47-57.

24 American Academy of Pediatrics, Committee on Hospital Care, Section on Surgery and Section on Critical Care. Policy statement: pediatric organ donation and transplantation. Pediatrics 2010; 125(4): 822-828.

25 Workman J, Myrick C, Meyers R, Bratton S, Nakagawa T. Pediatric organ donation and transplantation. Pediatrics 2013; 131(6): e1723-e1730.

26 Daley M, Limbo R. RTS Bereavement Training in Early Pregnancy Loss, Stillbirth \& Newborn Death, 2nd edn. Gundersen Lutheran Medical Foundation, Inc.: La Crosse, WI, USA, 2008.

27 Now I Lay Me Down to Sleep - NILMDTS, see www.NILMDTS.org

28 NICU Helping Hands. Available from www.nicuhelpinghands.org.

29 Wallerstedt C, Higgins P. Facilitating perinatal grieving between the mother and the father. J Obstet Gynecol Neonatal Nurs 1996; 25: 389-394.

30 Kenner C. Palliative and end-of-life care. In: Kenner C, Lott J (eds). Comprehensive Neonatal Nursing Care, 5th edn. Springer Publishing Company: New York, NY, 2014, pp 766-772.

31 Carnevale F, Alexander E, Davis M, Rennick J, Troini R. Daily living with distress and enrichment: the moral experience of families with ventilator-assisted children at home. Pediatrics 2006; 117(1): e48-e60.
32 Rider E, Volkan K, Halfer J. Pediatric residents' perceptions of communication competencies: implications for teaching. Med Teach 2008; 30(7): e208-e217.

33 Boss R, Hutton N, Donohue P, Arnold R. Neonatologist training to guide family decision making for critically ill infants. Arch Pediatr Adolesc Med 2009; 163(9): 738-788.

34 EPEC. EPEC: Education in Palliative and End-of-Life Care [Internet], 2013. Available from http://www.epec.net/.

35 End-of-Life Nursing Education Consortium (ELNEC). Available from http://www. aacn.nche.edu/ELNEC/2012.

36 Meadors $\mathrm{P}$, Lamson A. Compassion fatigue and secondary traumatization: provider self care on intensive care units for children. J Pediatr Health Care 2008; 22(1): 24-34.

37 Czaja A, Moss M, Mealer M. Symptoms of post-traumatic stress disorder among pediatric acute care nurses. J Pediatr Nurs 2012; 27(4): 357-365.

38 Boyle D. Countering compassion fatigue: a requisite nursing agenda. Online $J$ Issues Nurs 2011; 16: 2.

39 Boyle EM. Pain and compassion in the neonatal unit-a neonatologist's view. Neuro Endocrinol Lett 2004; 25(Suppl 1): 49-55.

40 Calhoun B, Napolitano P, Terry M. Perinatal hospice: comprehensive care for the family of the fetus with a lethal condition. J Reprod Med 2003; 48: 343-348.

41 Bennett J, Dutcher J, Snyders M. Embrace: addressing anticipatory grief and bereavement in the perinatal population: a palliative care case study. $J$ Perinat Neonatal Nurs 2011; 25(1): 72-76.

42 Rosenbaum J, Smith J, Zollfrank R. Neonatal end-of-life spiritual support care. J Perinat Neonatal Nurs 2011; 25(1): 61-69.

43 Carter B. Providing palliative care for newborns. Pediatr Ann 2004; 33(11): 770-777.

44 De Lisle-Porter M, Podruchny A. The dying neonate: family-centered end-oflife care. Neonatal Netw 2009; 28(2): 75-83.

45 National Association of Perinatal Social Workers. Standards for Social Work Services in Perinatal Bereavement [Internet]. National Association of Perinatal Social Workers, 2007. Available from http://napsw.org/about/pdfs/bereavementstandards.pdf.

46 Pearson L. Family-centered care and the anticipated death of a newborn. Pediatr Nurs 1997; 23(2): 178-182.

47 Levetown M, American Academy of Pediatrics Committee on Bioethics. Communicating with children and families: from everyday interactions to skill in conveying distressing information. Pediatrics 2008; 121(5): e1441-e1460.

48 Aladangady N, de Rooy L. Withholding or withdrawal of life sustaining treatment for newborn infants. Early Hum Dev 2012; 88(2): 65-69.

49 National Association of Neonatal Nurses. NICU Nurse Involvement in Ethical Decisions (Treatment Of Critically III Newborns). Position Statement \#3015. National Association of Neonatal Nurses: Chicago, IL, 2010. Available from http://www. nann.org/uploads/files/3015_reviewed_February_2010.pdf.

50 Bilgen H, Topuzoglu A, Kuscu K, Altuncu E, Ozek E. End-of-life decisions in the newborn period: attitudes and practices of doctors and nurses. Turk $J$ Pediatr 2009; 51(3): 248-256.

51 Craig F, Goldman A. Home management of the dying NICU patient. Semin Neonatol 2003; 8(2): 177-183.

52 Craig F, Mancini A. Can we truly offer a choice of place of death in neonatal palliative care? Semin Fetal Neonatal Med 2013; 18(2): 93-98.

53 Sanchez-Reilly S, Morrison S, Carey E, Bernacki R, O'Neil L, Kapo J et al. Caring for oneself to care for others: physicians and their selfcare. J Support Oncol 2013; 11 (2): 75-81.

54 Gold K, Dalton V, Schwent T. Hospital care for parents after perinatal death. Obstet Gynecol 2007; 109(5): 1156-1166

55 Sharp C. Use of the chaplaincy in the neonatal intensive care unit. South Med J 1991; 84(12): 1482-1486.

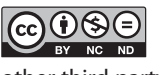

This work is licensed under a Creative Commons AttributionNonCommercial-NoDerivs 4.0 International License. The images or other third party material in this article are included in the article's Creative Commons license, unless indicated otherwise in the credit line; if the material is not included under the Creative Commons license, users will need to obtain permission from the license holder to reproduce the material. To view a copy of this license, visit http:// creativecommons.org/licenses/by-nc-nd/4.0/ 